Corresponding authors: desymer@mdanderson.org; william.carson@osumc.edu

C 2018 Suarez-Kelly et al. This article is distributed under the terms of the Creative Commons Attribution-NonCommercial License, which permits reuse and redistribution, except for commercial purposes, provided that the original author and source are credited.

Ontology terms: neoplasm of the breast; neurofibromas

Published by Cold Spring Harbor Laboratory Press

doi: 10.1101/mcs.a002352

\section{Metaplastic breast cancer in a patient with neurofibromatosis type 1 and somatic loss of heterozygosity}

\author{
Lorena P. Suarez-Kelly, ${ }^{1}$ Keiko Akagi, ${ }^{1,2,4}$ Julie W. Reeser, ${ }^{1}$ Eric Samorodnitsky, ${ }^{1}$ \\ Matthew Reeder, ${ }^{1}$ Amy Smith, ${ }^{1}$ Sameek Roychowdhury, ${ }^{1}$ David E. Symer, ${ }^{1,2,5}$ \\ and William E. Carson ${ }^{1,3}$

\begin{abstract}
${ }^{1}$ The Arthur G. James Comprehensive Cancer Center and Richard J. Solove Research Institute, The Ohio State University, Columbus, Ohio 43210, USA; ${ }^{2}$ Department of Cancer Biology and Genetics, The Ohio State University, Columbus, Ohio 43210, USA; ${ }^{3}$ Division of Surgical Oncology, Department of Surgery, The Ohio State University, Columbus, Ohio 43210, USA
\end{abstract}

\begin{abstract}
Metaplastic breast carcinoma (MBC) is rare and has a poor prognosis. Here we describe genetic analysis of a 41-yr-old female patient with $M B C$ and neurofibromatosis type I (NF1). She initially presented with pT3N1a, grade 3 MBC, but lung metastases were discovered subsequently. To identify the molecular cause of her NF1, we screened for germline mutations disrupting NF1 or SPRED1, revealing a heterozygous germline single-nucleotide variant (SNV) in exon 21 of NF1 at c.2709G>A, Chr 17: 29556342. By report, this variant disrupts pre-mRNA splicing of NF1 transcripts. No pathogenic mutations were identified in SPRED1. A potential association between MBC and NF1 was reported in eight previous cases, but none underwent detailed genomics analysis. To identify additional candidate germline variants potentially predisposing to $\mathrm{MBC}$, we conducted targeted exome sequencing of 279 established cancer-causing genes in a control blood sample, disclosing four rare SNVs. Analysis of her breast tumor showed markedly altered variant allelic fractions (VAFs) for two (50\%) of them, revealing somatic loss of heterozygosity $(\mathrm{LOH})$ at germline SNVs. Of these, only the VAF of the pathogenic SNV in NF1 was increased in the tumor. Tumor sequencing demonstrated five somatic mutations altering TP53, BRCA1, and other genes potentially contributing to cancer formation. Because somatic $\mathrm{LOH}$ at certain germline SNVs can enhance their impacts, we conclude that increased allelic imbalance of the pathogenic SNV in NF1 likely contributed to tumorigenesis. Our results highlight a need to assess predisposing genetic factors and $\mathrm{LOH}$ that can cause rare, aggressive diseases such as MBC in NF1.
\end{abstract}

[Supplemental material is available for this article.]

\section{INTRODUCTION}

Metaplastic breast carcinoma $(\mathrm{MBC})$ is rare, comprising $<1 \%$ of all breast cancers (Natsiopoulos et al. 2007; Toumi et al. 2011; Cooper et al. 2013; Roy et al. 2014). MBC is comprised of a heterogeneous group of cancers consisting of glandular epithelial cell and mixed nonglandular epithelial or mesenchymal cell types (Greenberg et al. 2004;

\footnotetext{
${ }^{4}$ Present address: Department of Thoracic/Head and Neck Medical Oncology, University of Texas MD Anderson Cancer Center, Houston, Texas 77030, USA

${ }^{5}$ Present address: Department of Lymphoma and Myeloma, University of Texas MD Anderson Cancer Center, Houston, Texas 77030, USA
} 
Natsiopoulos et al. 2007; Toumi et al. 2011). This form of breast cancer usually is associated with advanced patient age, lower incidence of axillary lymph node involvement, larger tumor size at time of diagnosis, advanced disease at time of diagnosis, infrequent hormone receptor expression (ER/PR), and rare overexpression of HER2/neu (Barnes et al. 2005). In comparison with more typical breast carcinomas, $\mathrm{MBC}$ has a worse disease-free survival and decreased overall survival (Luini et al. 2007). A study comparing a large cohort of patients with $\mathrm{MBC}$ to patients with triple-negative invasive ductal carcinoma, who also have a poor prognosis, found that disease-free survival and overall survival were even worse in the patients with MBC (5-yr rates, $45.6 \%$ vs. $81.6 \% ; P<0.001$, and $53.7 \%$ vs. $84.6 \% ; P<0.001$, respectively) (Lee et al. 2012). On univariate and multivariable analyses, MBC was found to be an independent prognostic factor for disease recurrence and overall survival (Lee et al. 2012).

Neurofibromatosis type I (NF1), also called von Recklinghausen's disease, is an inherited genetic disorder with autosomal dominant inheritance. Its incidence has been reported to be $\sim 1$ out of 2500-4000 live births, whereas most population-based studies have demonstrated its prevalence as 1 in 2000 to 1 in 5000 individuals (Salemis et al. 2010; Madanikia et al. 2012; Campos et al. 2013; Lakshmaiah et al. 2014; Sobjanek et al. 2014; Xu et al. 2014). NF1 is a fully penetrant condition, with all affected individuals developing at least some of the characteristic neurocutaneous features. These include neurofibromas, café au lait spots, axillary or groin freckling, optic pathway glioma, heritability, and eye conditions such as hamartomas of the iris (i.e., Lisch nodules) (Sharif et al. 2007; Vivas et al. 2014; Xu et al. 2014). In addition to these features, other common phenotypes may include cognitive impairment, vasculopathies, and skeletal abnormalities including macrocephaly, scoliosis, bony dysplasia of long bone or sphenoid, short stature, pectus excavatum, and pseudoarthrosis of the tibia (Brasfield and Das Gupta 1972; Huson et al. 1988; Ferner et al. 2007; Boyd et al. 2009; Madanikia et al. 2012). A related, NF1-like syndrome that shares many features with NF1 has been characterized recently and is called Legius syndrome (Brems et al. 2007).

The gene that primarily is mutated in and responsible for NF1 is neurofibromin 1 (NF1), located on human Chromosome 17q11.2. The gene spans more than $280 \mathrm{~kb}$ and contains 62 exons. Transcripts (e.g., RefSeq NM_000267.3) encode neurofibromin, a regulator of the RAS-MAPK signaling pathway (Korf 2013; Xu et al. 2014). Neurofibromin functions as a tumor suppressor through inhibition of RAS activation (Sharif et al. 2007; Xu et al. 2014). NF1 has one of the highest reported mutation rates in the human genome, with more than 1400 distinct mutations reported in the literature (Xu et al. 2014). A second gene, SPRED1, has been implicated in pathogenesis of Legius syndrome (Brems et al. 2012).

Mutations in NF1 disrupt neurofibromin function, resulting in downstream cell activation and predisposition to the development of several types of cancers (Sharif et al. 2007; Xu et al. 2014). The most commonly reported NF1-specific malignancies are intracranial gliomas and malignant peripheral nerve sheath tumors. Additional, common NF1-related cancers include breast, mouth or pharynx, thyroid, and eye tumors (Zöller et al. 1997; Sharif et al. 2007; Salemis et al. 2010; Madanikia et al. 2012; Khalil et al. 2015; Uusitalo et al. 2017). Gastrointestinal stromal tumors and pheochromocytomas also have been associated with NF1 (Walther et al. 1999; Maertens et al. 2006). The most commonly reported type of breast cancer in the context of NF1 is invasive ductal carcinoma (Vivas et al. 2014).

NF1 has been implicated as a breast cancer driver gene, with somatic mutations reported in $28 \%$ of all breast carcinomas (Wallace et al. 2012). Somatic loss of NF1 has also been reported in malignant phyllodes tumors (Lee et al. 2010). In addition, its epigenetic silencing has been correlated with tamoxifen resistance in breast cancer (Mendes-Pereira et al. 2012). A mutation in NF1 may also result in, or predispose cells to, a secondary mutation in other genes on the same chromosome (i.e., on Chr 17) (Yap et al. 2014).

Somatic loss of heterozygosity $(\mathrm{LOH})$ of genetic variants affecting cancer-causing genes, particularly tumor suppressor genes, has been observed to occur frequently in many human 
cancers including breast cancers (Deng et al. 1996; Kerangueven et al. 1997; The Cancer Genome Atlas Network 2012; Gao et al. 2013). LOH can result in reduced copy number of the wild-type genotype, thereby increasing the relative impact of a deleterious genotype. $\mathrm{LOH}$ or allelic imbalance has been described in normal-appearing breast epithelial tissue adjacent to the tumor itself, suggesting that it may precede or contribute to cancer formation (Larson et al. 2005).

Here we report the results of a detailed molecular analysis of a patient with clinical NF1 who presented with MBC. Using targeted next-generation sequencing, we identified genetic variants in the patient's blood (germline) and/or in her tumor (somatic tissue) that likely caused her NF1 and contributed to her MBC. A systematic review of the existing literature regarding MBC in patients with NF1 revealed several previous case reports, but none described use of modern genomics methods to identify contributing genetic alterations.

\section{RESULTS}

\section{Clinical Presentation}

The patient is a 41-yr-old G3P3 female who presented to her primary care provider with a large mass in her right breast. She reported that her onset of menarche was at $13 \mathrm{yr}$ of age, and her first pregnancy occurred at $20 \mathrm{yr}$ of age. Her past medical history was significant for hypertension and leukemia (treated as a child). Her family history was notable for cancers in her paternal aunt, paternal grandmother, paternal grandfather, and paternal great-grandmother, although the specific types of cancer were not known. Diagnostic mammography demonstrated a smoothly marginated, homogeneously dense 4-cm mass in the upper outer quadrant of the right breast. An ultrasound disclosed a $6.3 \times 3.8 \times 3.6-\mathrm{cm}$, complex cystic mass with internal echogenic material. The mass was biopsied, revealing a malignant spindle cell neoplasm with necrosis.

About 10 wk later, she was seen at The Ohio State University Comprehensive Cancer Center for further evaluation by breast surgeons. She complained of progressive enlargement of her breast mass. Physical examination revealed a mobile, nontender, $\sim 10-\mathrm{cm}$ mass in the right breast, centered in the upper quadrant with thinning of the overlying skin (Fig. 1A). No nipple retraction or discharge was noted, and there was no palpable axillary adenopathy.

We documented three cardinal features of the clinical syndrome NF1, exceeding a requirement for at least two NIH criteria for the diagnosis (Ferner et al. 2007). These included (a) axillary and groin freckling, and (b) at least a dozen skin lesions on her chest wall considered likely neurofibromas (Fig. 1A). A biopsy of one of these was confirmed cutaneous neurofibroma upon pathologic diagnosis. The patient also had been informed previously that (c) she and her two sons had NF1 (i.e., a genetic disease involving café au lait spots). In addition, two large (>15-mm) café au lait spots were observed, including one on her right torso, but this count was insufficient to qualify as a fourth diagnostic criterion for NF1 (Ferner et al. 2007). We were unable to evaluate other $\mathrm{NIH}$ criteria for NF1 in the patient.

Repeat mammography demonstrated a $12 \times 11 \times 7-\mathrm{cm}$ mass in the outer quadrant of the right breast, with new calcifications and a few smaller masses inferior and medial to it (Fig. 1B, C). Ultrasonography showed a large complex mass with areas of internal vascularity, and the satellite nodules seen on mammography appeared to be extensions of the dominant mass. It measured $11 \times 5 \times 7 \mathrm{~cm}$, indicating a tumor doubling time of $38 \mathrm{~d}$ (Fig. 1D,E). Further staging was performed by computed tomographic (CT) imaging of the chest, abdomen, and pelvis, which identified a large necrotic right breast mass, multiple small indeterminate pulmonary nodules, bilateral indeterminate adrenal nodules, and no evidence of bony metastases (not shown). 

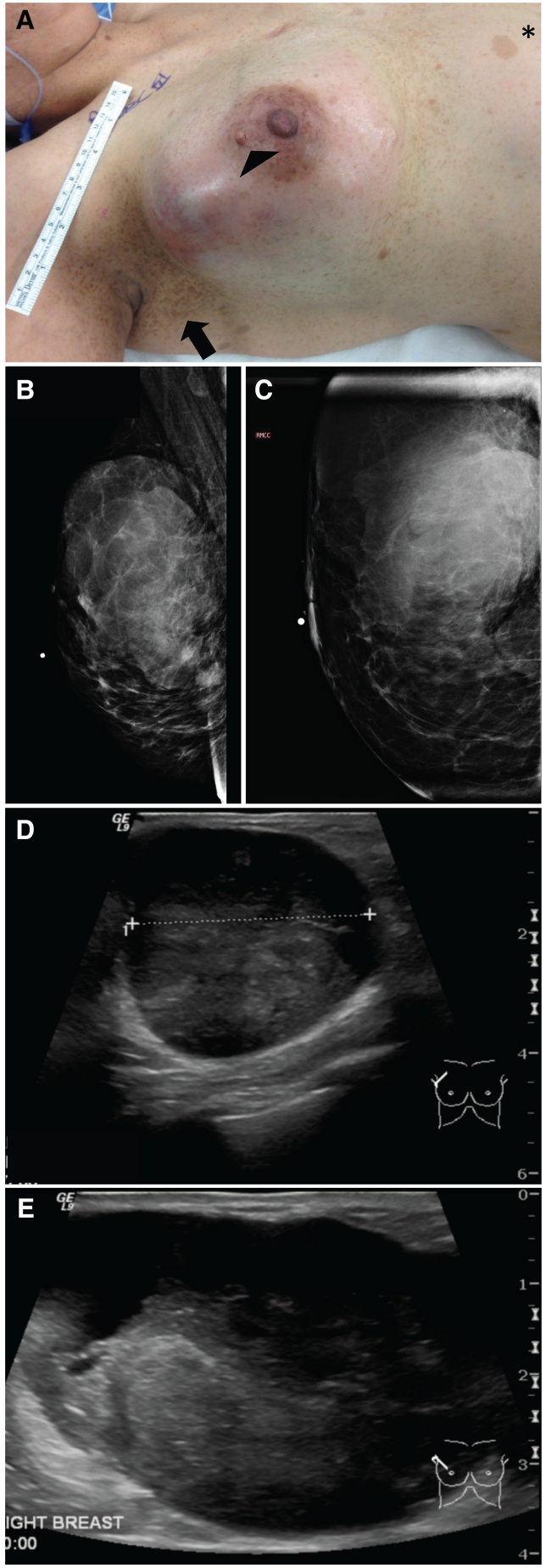

Figure 1. Clinical presentation of a patient with MBC and NF1. (A) Clinical presentation. A 41-yrold female with NF1 presented with a right breast mass diagnosed as MBC. On physical examination the breast mass was mobile, nontender, and $\sim 10$ $\mathrm{cm}$ in diameter with thinning of the overlying skin. She was also noted to have subtle features of NF1, including a café au lait spot on the right torso $(*)$, axillary and groin freckling (arrow), and a skin lesion resembling a neurofibroma (arrowhead). Diagnostic mammography: $(B)$ mediolateral and $(C)$ craniocaudal spot magnification mammogram views of the right breast demonstrate a dense mass in the upper outer quadrant with associated calcifications measuring $12 \times 11 \times 7 \mathrm{~cm}$. $(D, E)$ Diagnostic ultrasonography. Sonographic images of the right breast demonstrate a large complex mass measuring $11 \times 7 \times 5 \mathrm{~cm}$ with internal echogenic material and faint areas of internal vascularity.

The patient underwent a right total mastectomy with sentinel lymph node biopsy followed by immediate reconstruction. Tumor pathology documented a $10.2-\mathrm{cm}$, grade 3 , pT3 pN1a, invasive MBC with focal squamous differentiation and extensive necrosis. The tumor was negative for estrogen receptor (ER), progesterone receptor (PR), and human 
epithelial growth factor receptor 2 (HER2) expression. Immunohistochemical staining demonstrated that the tumor was positive for p40, cytokeratin (CK) 5/6, and AE1/AE3, and negative for Cam 5.2, supporting the diagnosis of MBC. The skin lesion was confirmed as a neurofibroma on final pathology. Cells stained positive for S100 and CD34 but negative for epithelial membrane antigen and smooth muscle actin.

The patient did well initially after surgery, and her incision healed well. She was seen by a medical oncologist for consideration of adjuvant therapy and was started on a regimen of carboplatin and paclitaxel. However, lung metastases were noted just 21 wk after surgery. She subsequently was treated by her local medical oncologist. Her current status is unknown.

\section{Molecular Basis for NF1 Disease}

To identify the genetic variant(s) that resulted in clinically diagnosed NF1, we performed targeted exome sequencing of the patient's normal blood, focusing on 279 known, cancercausing genes (Supplemental Table 1). The hybrid capture panel included exons from NF1 isoform 2, so potential heterozygous mutations causing the clinical syndrome NF1 could be detected. Counts of sequence reads that were obtained and aligned to the targeted exome regions, and other summary statistics of targeted sequencing, are presented in Supplemental Table 2.

The targeted exome-sequencing data revealed eight single-nucleotide variants (SNVs) in NF1, each present in heterozygosity in the normal blood sample (Fig. 2; Supplemental Table 3). Using algorithms designed to identify deleterious SNVs, each of these initially was predicted to be benign, either because they were synonymous variants or because they were noncoding.

Out of the eight SNVs in NF1, a heterozygous G>A substitution in codon 2709, exon 21 at Chr 17: 29556342 (referred to as NF1;c.2709G>A;[=], based on NF1 reference transcript NM_000267.3) was noted to occur extremely rarely in healthy donor populations (Table 1; Fig. 2F; Supplemental Table 3). Although this particular NF1 variant is synonymous and therefore was predicted to be a benign SNV, it has been reported to cause pre-mRNA mis-splicing by creating a cryptic alternative splice donor site in exon 21 in the NM_00267.3 mRNA reference sequence (Brinckmann et al. 2007; Wimmer et al. 2007). This would result in deletion of $144 \mathrm{nt}$ from the expressed transcript (i.e., r.2707 2850del144) and 48 amino acids from the translated protein (i.e., p.Val903_ Gln950del48), thereby disrupting the gene's expression and function. The same variant was previously identified in other patients meeting the NIH diagnostic criteria for NF1 (Brinckmann et al. 2007; Wimmer et al. 2007). This SNV (rs771820789) also was reported as pathogenic for NF1 by Ambry Genetics (RCV000492711) and as pathogenic or likely pathogenic for NF1 by the Centre for Mendelian Genomics, University Medical Centre Ljubljana and Invitae (RCV000415263). We confirmed the same SNV in our patient using two other molecular methods: that is, Haloplex sequencing and Sanger sequencing (performed and reported by the Medical Genomics Laboratory at University of Alabama, Birmingham [UAB]; data not shown). Thus we attributed the patient's NF1 to this heterozygous germline variant in NF1.

To exclude the possibility that the patient's NF1 was caused by germline mutations in SPRED1, which cause Legius syndrome (Messiaen et al. 2009), we also screened for mutations in it. However, no pathogenic SNVs were identified in SPRED1 using Haloplex sequencing (performed and reported by UAB; data not shown).

\section{Identification of Candidate Cancer-Predisposing Genetic Variants}

To identify additional germline SNVs that could predispose to MBC in the context of NF1, we examined data from targeted exome capture sequencing from the patient's blood sample. A 
Downloaded from molecularcasestudies.cshlp.org on April 26, 2023 - Published by Cold Spring Harbor Laboratory Press
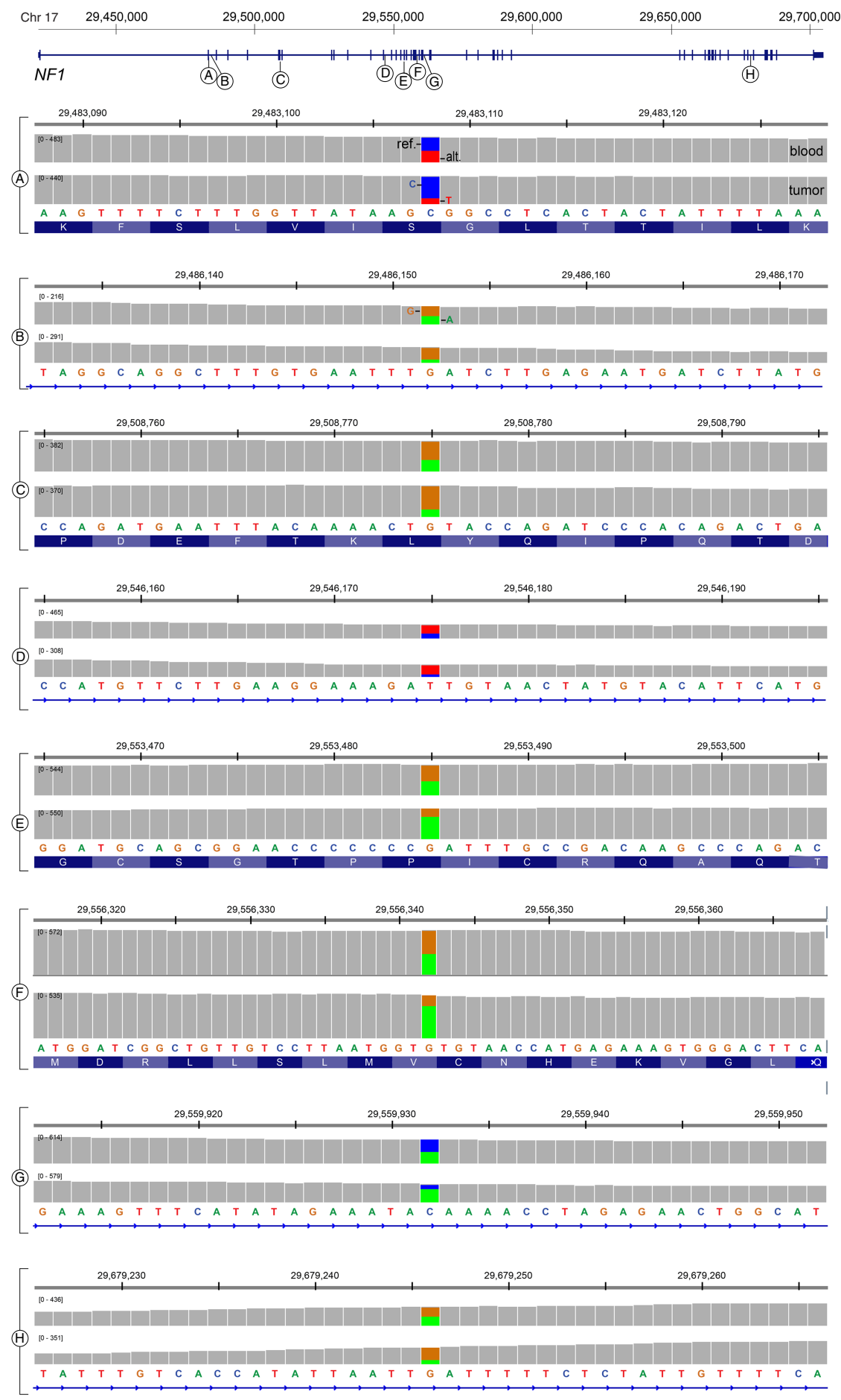

Figure 2. Targeted exome sequencing reveals SNVs in NF1. (Top) schematic of the NF1 gene locus on Chr 17 including coordinates (based on hg19 reference genome), positions of exons (blue rectangles), and positions of SNVs. (A-H) (Top) Shown here for each SNV are integrated genome viewer (IGV; Broad Institute) displays of aligned, targeted exome sequences including SNVs. Middle (above) normal germline and (below) MBC tumor sample sequence counts at each identified position. Gray bars, the height of each bar is related to counts of aligned sequences matching the reference genome; colored bars in center, relative counts of (top) reference (ref.) and (bottom) variant (alt.) sequence reads, respectively. For each SNV, reference allele sequence counts are displayed on the top of the histogram and the alternate allele on the bottom. Each base is depicted by a representative color: green, A; red, T; blue, C; orange/brown, G. (Bottom) Reference genome sequence and (blue rectangles) encoded amino acids or (small blue arrows, horizontal blue lines) introns. 
total of 339 germline SNVs involving 141 of the 279 targeted genes were identified (Supplemental Table 3) using SAMtools mPileup software analysis.

Of these SNVs, four were identified as potentially impactful variants, based on two criteria: They were predicted to disrupt transcript splicing or alter an amino acid, and they occur rarely in healthy control populations (Table 1; Supplemental Table 3). Particular variants predicted by variant effect predictor (VEP) software to cause missense or splicing region changes were tabulated. In addition, we hypothesized that rare germline SNVs (i.e., each with a population allele frequency of $<1 \%$ in each of the 1000 Genomes phase 3, gnomAD, and ESP AD populations) would be expected to exert greater functional impacts than more common alleles, because the latter would have survived purifying selection in a healthy population (Table 1; Supplemental Table 3; Kryukov et al. 2007). Included in this list of candidate functional SNVs was the rare NF1 variant that disrupts transcript splicing. We referred to this list of germline SNVs as those with potential functional impacts. They could predispose to cancer development, by disrupting the expression, structure, or function of BRCA2, CSF1R, NF1, and STAT1 (Table 1). Additional predictions of potential impacts on encoded proteins' structure and function were tabulated using SIFT, PolyPhen, MetaSVM, Condel, and CADD (Phred score) by VEP. Annotated clinical impacts were tabulated using ClinVar (Supplemental Table 3).

Focusing on the possible functional impacts of the six germline SNVs detected in BRCA2 (Supplemental Table 3; Supplemental Fig. 1), we determined their population allele frequencies, predicted impacts on protein expression and structure, and status in databases tabulating the known, predisposing determinants of breast cancer. Of these six SNVs, one at Chr 13:32945172 (rs11571747, ENST00000544455.1:c.8567A>C) was a rare, missense mutation, occurring at a population allele frequency of $<1 \%$, and therefore was included

Table 1. Functional single-nucleotide variant (SNV) candidates: germline SNVs that are both rare and potentially deleterious

\begin{tabular}{|c|c|c|c|c|c|c|c|c|c|}
\hline Chr & Coordinate & Gene & ref. & var. & \multicolumn{2}{|c|}{$\begin{array}{l}\text { Variant } \\
\text { identifier }\end{array}$} & \multicolumn{2}{|c|}{ Predicted amino acid change } & $\begin{array}{l}\text { Pred. } \\
\text { conseq. }\end{array}$ \\
\hline 2 & 191850338 & STAT1 & C & $\mathrm{T}$ & \multicolumn{2}{|c|}{ rs56228116 } & \multicolumn{2}{|c|}{ Splicing region } & SP \\
\hline 5 & 149433888 & CSF1R & C & G & \multicolumn{2}{|c|}{ rs34030164 } & \multicolumn{2}{|c|}{ ENSP00000286301.3:p.Glu920Asp } & M \\
\hline 13 & 32945172 & BRCA2 & A & C & rs11 & 571747 & \multicolumn{2}{|c|}{ ENSP00000439902.1:p.Glu2856Al } & Ala \\
\hline 17 & 29556342 & NF1 & G & A & rs77 & 1820789 & \multicolumn{2}{|c|}{ ENSP00000351015.4:p.Val903= } & SYN \\
\hline \multicolumn{10}{|l|}{$B$} \\
\hline Chr & Coordinate & Gene & \multicolumn{3}{|c|}{$\begin{array}{c}1000 \text { Genomes } \\
\text { (VEP r.90) }\end{array}$} & gnomAD & ESP_EA & $\begin{array}{l}\text { ClinVar clinical } \\
\text { significance }\end{array}$ & $\begin{array}{l}\text { Change in } \\
V A F \text { in tumor }\end{array}$ \\
\hline 2 & 191850338 & STAT1 & \multicolumn{3}{|c|}{0.0002} & 0.000398 & 0.001163 & Likely benign & 0.0505 \\
\hline 5 & 149433888 & CSF1R & \multicolumn{3}{|c|}{0.001} & 0.002729 & 0.002558 & Likely benign & -0.068 \\
\hline 13 & 32945172 & BRCA2 & \multicolumn{3}{|c|}{0.0002} & 0.001073 & 0.001977 & $\begin{array}{l}\text { Benign and likely } \\
\text { benign }\end{array}$ & -0.2706 \\
\hline 17 & 29556342 & NF1 & \multicolumn{3}{|c|}{ NA } & NA & NA & Likely benign & 0.2725 \\
\hline
\end{tabular}

$(A, B)$ Of 339 germline SNVs detected from targeted exome sequencing of the patient's normal blood sample, we identified four having $<1 \%$ population allele frequency and predicted consequences. Chr, chromosome (hg19 human genome reference assembly); ref., reference allele; var., variant allele; variant identifier, dbSNP accession number; predicted amino acid change and p., ENSEMBL accession number and amino acid position; pred.cons., predicted consequence of (SP) splicing region, (M) missense or (SYN) synonymous changes in the transcribed pre-mRNA. (B) 1000 Genomes, gnomAD, ESP AD, population allele frequencies in Variant Effect Predictor release 90; ClinVar, curated clinical consequences; change in VAF in tumor, calculated as the SNV's VAF in the tumor-its VAF in normal blood; NA, SNV not observed in healthy population variant database. Highlighted yellow, germline SNV in NF1 that is synonymous but creates a cryptic pre-mRNA splicing donor site and results in NF1. See Supplemental Table 3 for further details. 
in our list of functional SNV candidates (Table 1). However, its clinical significance was reported as benign or likely benign in the ClinVar database. This same SNV also was shown to be neutral (Spearman et al. 2008). The other five germline variants in BRCA2 were excluded from the list of functional SNV candidates because of their relatively high population allele frequencies (>1\%).

\section{Tumor-Specific Somatic Variants}

We used MuTect at default settings to identify a total of five somatic SNVs (mutations) in the patient's MBC sample (Table 2; Supplemental Table 4). Each was supported by at least $5 \%$ of the sequence reads aligned to the variant position in the tumor genome. The five potentially deleterious, somatic SNVs were identified in VEGFA, TSC2, TP53, BRCA1, and VHL. Four of the five SNVs were nonsynonymous and therefore potentially deleterious to gene function. The fifth SNV was an intronic variant in VHL and potentially could disrupt pre-mRNA splicing. Because the variant allelic fraction (VAF) of each of these somatic SNVs was $<50 \%$, we concluded that they represented a subclonal population of tumor cells harboring the mutant allele. However, based on these data alone, we could not determine the relative ploidy or the purity of the tumor cells.

The somatic variant identified in TP53 was previously cataloged as likely to be pathogenic as per the ClinVar database (Supplemental Table 4). Annotations for the somatic variant in $B R C A 1$ showed conflicting functional impacts, and therefore its potential pathogenic significance in contributing to MBC in this patient remained uncertain. The variant in TSC2 was not identified in the ClinVar clinical significance database, but it was listed in the ExAC database as an extremely rare germline variant. The intronic variant in VHL has been annotated in $\mathrm{dbSNP}$, but it and the novel (unannotated) SNV in VEGFA were not listed in the ClinVar database (Table 2).

\section{Somatic Loss of Heterozygosity}

To investigate the possibility that somatic $\mathrm{LOH}$ or allelic imbalance could increase functional impacts of particular germline SNVs in the tumor, we calculated the VAFs for each of the 339 germline SNVs detected, in both the normal (blood, germline) DNA (Fig. 3A) and tumor (somatic) samples (Fig. 3B). We then calculated the difference between the tumor VAF and matched normal sample VAF for each germline SNV identified (Fig. 3C), as well as the absolute value of these differences (Fig. 3D). These VAF differences showed a clear bimodal distribution (Fig. 3C,D), suggesting that the VAF for many SNVs did not change between

Table 2. Somatic variants detected in the tumor

\begin{tabular}{lcccccccc}
\hline Chr & Coordinate & ref. & var. & Gene & $\begin{array}{c}\text { Change in amino } \\
\text { acid }\end{array}$ & $\begin{array}{c}\text { SNV } \\
\text { annotation }\end{array}$ & $\begin{array}{c}\text { Variant allele } \\
\text { fraction }\end{array}$ & ClinVar clinical significance \\
\hline 3 & $10,188,141$ & G & A & VHL & NA & rs541565291 & 0.068 & NA \\
6 & $43,738,969$ & C & T & VEGFA & p.R176W & NA & 0.247 & NA \\
16 & $2,130,253$ & C & T & TSC2 & p.P1162L & rs778069675 & 0.303 & NA \\
17 & $7,577,548$ & C & T & TP53 & p.G245S & CM010463 & 0.411 & Likely pathogenic \& pathogenic \\
17 & $41,197,778$ & A & T & BRCA1 & p.W1858R & rs80356959 & 0.355 & Uncertain significance \& not provided \& \\
& & & & & & & & likely pathogenic \\
\hline
\end{tabular}

To compare variant calls with the matched normal DNA, we used MuTect at default settings. Tabulated here are the chromosomal coordinates: ref., reference; var., variant of nucleotide substitution; involved gene; predicted coding change in protein amino acid; annotation of the SNV in dbSNP or COSMIC databases; calculated VAF in the tumor; and clinical significance as documented in the ClinVar database. NA, not annotated in database. See Supplemental Table 4 for further details. 

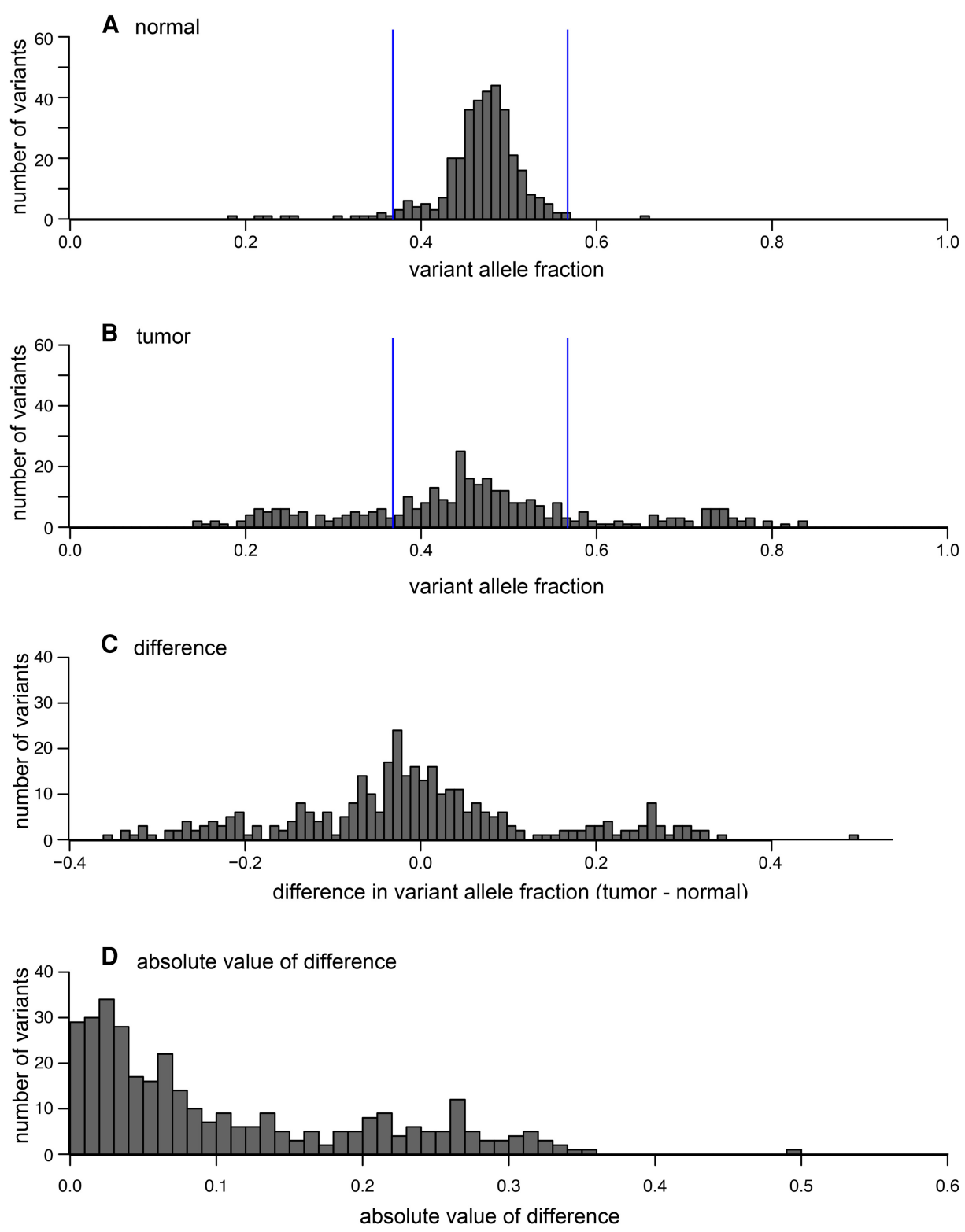

Figure 3. Variant allele fractions of SNVs in normal and tumor samples. $(A, B)$ Shown here are the distributions of VAF counts for all $339 \mathrm{SNV}$ s detected in $(A)$ normal germline and $(B)$ tumor samples. For each SNV, the VAF was calculated in each sample as the ratio of the number of sequence reads documenting the alternate allele versus all sequence reads aligned to that position. (Vertical blue lines) Limits of the distribution falling within three standard deviations of the median VAF values. (C) A histogram showing the trimodal distribution of the differences between tumor VAFs and normal sample VAFs. (D) The absolute value of the difference between tumor VAFs and normal sample VAFs, showing a bimodal distribution.

tumor and matched normal sample. However, other SNVs showed a large increase or decrease in their tumor VAF relative to the matched normal control.

Additional examples of SNVs detected in genes both in the normal (blood, germline) and tumor (somatic) samples are shown in Supplemental Figure 2. Included in this arbitrarily chosen set of SNVs are examples where somatic LOH resulted in the loss of either the variant or the reference allele in the tumor, respectively. In one case, a somatic SNV and a germline 


\begin{tabular}{lcccc}
\hline \multicolumn{5}{l}{ Table 3. Genes with multiple instances of somatic loss of heterozygosity (LOH) } \\
\hline Gene & $\begin{array}{c}\text { No. germline } \\
\text { SNVs }\end{array}$ & $\begin{array}{c}\text { No. SNVs with significant } \\
\text { change in VAF in tumor }\end{array}$ & $\begin{array}{c}\text { Frequency of SNVs } \\
\text { with LOH (\%) }\end{array}$ & $\begin{array}{c}\text { Range of absolute } \\
\text { changes in VAFs (\%) }\end{array}$ \\
\hline NF1 & 8 & 7 & 87.5 & $13.3-28.6$ \\
BRCA2 & 6 & 6 & 100 & $21.4-34.7$ \\
TNKS & 6 & 6 & 100 & $20.7-30.5$ \\
ATM & 4 & 4 & 100 & $16.0-29.9$ \\
EPHA7 & 4 & 4 & 100 & $23.6-30.3$ \\
IGF2R & 4 & 4 & 100 & $20.0-31.3$ \\
RPTOR & 4 & 4 & 100 & $31.1-49.1$ \\
WT1 & 4 & 4 & 100 & $19.7-35.3$ \\
EZH2 & 3 & 3 & 100 & $21.0-29.9$ \\
PTPRD & 3 & 3 & 100 & $21.9-27.5$ \\
RPA1 & 3 & 3 & 100 & $21.6-32.1$ \\
\hline
\end{tabular}

Listed here are genes targeted by our exome capture panel that harbor at least three germline single-nucleotide variants $(\mathrm{SNV}$ ), of which at least 80 showed significantly altered variant allele fractions (VAFs) in the tumor. Significant changes in individual VAFs were defined as absolute increases or decreases of at least 15 in the tumor, respectively. The range of absolute changes in VAFs (by comparing the same SNVs in germline vs. tumor samples) is presented for each gene. See Supplemental Table 3 for further information.

SNV were detected within an 8-nt genomic interval of each other in VEGFA (Supplemental Fig. 2B). This provided a useful internal control to compare their VAFs in the blood versus tumor sample. The germline variant did not show evidence of somatic $\mathrm{LOH}$ in the tumor, because its VAF was not significantly changed from $50 \%$ when compared with the normal blood sample. In contrast, the VAF at the somatic SNV was essentially 0\% in the germline sample as expected, and $<50 \%$ in the tumor, suggesting that it was not detected in every cell present in the tumor sample. This showed that either the tumor was not only comprised of tumor cells (i.e., normal plus tumor cell genomes would lead to this outcome) or clonal heterogeneity existed in the tumor.

Of the 141 genes in which germline SNVs were identified, 11 genes were found to harbor three or more SNVs each, where $>80 \%$ of the SNVs showed significant changes in the tumor VAF (Table 3). NF1 was at the top of this list of genes; seven out of eight of its germline heterozygous variants showed significant changes in their VAFs in the tumor. This demonstrated that a significant level of somatic LOH was observed for most or all of the SNVs detected in these several genes, suggesting that they each gained or lost a long haplotype or allele in the tumor.

Our panel of cancer-causing gene exomes was not designed to represent or sample all genes or chromosomes uniformly, genome-wide. Nevertheless, to characterize how LOH could affect various genes or chromosomes differentially, we plotted the VAF for each SNV identified in the patient's normal blood. The results showed that somatic LOH differentially affected many or most SNVs localized on particular chromosomes including Chr 4, 13, and 17 (Fig. 4). Notably, NF1 is located on Chr 17q (Fig. 2), and BRCA2 is located on Chr 13 (Supplemental Fig. 1), where apparent chromosome-wide LOH was observed. Interestingly Chr 17q was identified as the only chromosomal arm that also was found to be significantly lost in normal breast epithelium in BRCA1 mutation carriers (Larson et al. 2005).

\section{Prediction of Functional Impacts by Germline SNVs with LOH}

Many of the 339 germline SNVs detected by our exome capture methods (Supplemental Table 2) showed somatic LOH in the tumor when compared to the matched normal sample 

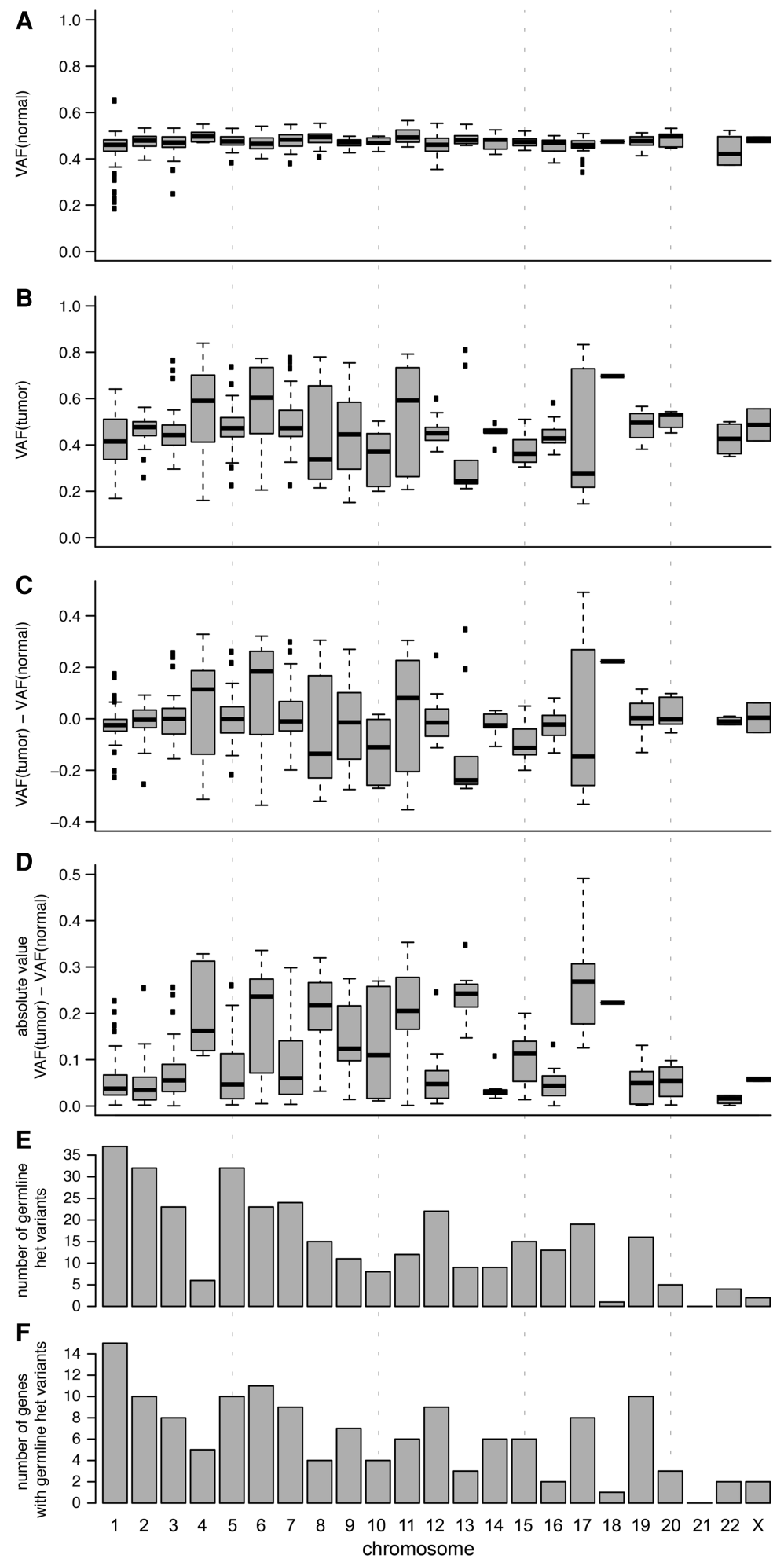

Figure 4. $\mathrm{LOH}$ is enriched at particular chromosomes. Shown here are box and whisker plots of the distribution of ( $y$-axis) SNV VAFs for each chromosome (x-axis) in the $(A)$ normal and $(B)$ tumor samples. $(C)$ The distribution of the difference between tumor VAFs and normal VAFs for each SNV is shown for each chromosome. Positive values, VAFs are increased in the tumor; negative values, VAFs are decreased in the tumor. (D) The absolute value of the differences between tumor VAFs and normal VAFs is shown for each SNV on the chromosomes. (E) Histogram of SNV counts as detected by exome capture sequencing is shown per chromosome. (F) Histogram of genes harboring the detected SNVs is shown per chromosome. 
(Figs. 3 and 4; Supplemental Table 3). The particular SNV in NF1 that is associated with altered pre-mRNA splicing of that gene showed $\mathrm{LOH}$, as the reference allele was almost completely lost, whereas the variant allele was enriched significantly in the tumor (VAF = 0.74; Fig. 2F; Supplemental Table 3). The directionality of the LOH increased the allelic imbalance of this particular variant (alternate) allele, so its functional impact thereby would increase in the tumor.

Based on this example of the disease-causing SNV in NF1, we hypothesized that changes in the VAFs of other functional SNV candidates (Table 1) could be used to corroborate their predicted functional impacts. If an alternate variant showed increased allelic imbalance in the tumor, resulting in hemizygosity of the variant, the variant allele could exert increased functional impact in the tumor because of decreased relative expression of the wild=type (reference) allele (Spearman et al. 2008).

One of the SNVs detected in BRCA2 was included in the list of functional variant candidates, as it was a rare, missense variant (Table 1; Supplemental Fig. 1). However, the VAF of the alternate allele was decreased in the tumor, suggesting consequential reductions in its potential functional impacts in the tumor. Consistent with this observation, this SNV has been cataloged as clinically benign. For these reasons, we conclude that it is an unlikely contributor to breast cancer.

The other functional SNV candidates (aside from the one pathogenic splicing variant in NF1) did not show significant changes in their VAFs in the tumor (Table 1; Supplemental Fig. 2). This showed that they did not undergo $\mathrm{LOH}$ and strongly suggested that their potential contributions to the malignant phenotype again were likely to be minimal.

\section{DISCUSSION}

Here we have presented a first-of-its-kind, molecular case report of a patient with MBC arising in the context of clinically diagnosed neurofibromatosis type 1 (NF1). NF1 is a cancerpredisposing syndrome, in addition to other clinical features outlined above. It usually is caused by a germline mutation that disrupts function of the tumor suppressor gene NF1. However, mutations in NF1 have been identified in only $78 \%$ of patients who fulfill NIH diagnostic criteria (Xu et al. 2014). Additional NF1-like cases have been attributed to variants in SPRED1. As we described here, targeted sequencing disclosed a rare, synonymous SNV in NF1 that has been reported to disrupt its pre-mRNA splicing, causing the patient's NF1.

NF1 patients have a fourfold increase $(95 \% \mathrm{Cl} 2.8-5.6)$ in their relative risk of malignancy (Sørensen et al. 1986; Rasmussen et al. 2001; Evans et al. 2011). A prospective study evaluating a cohort of 448 NF1 patients reported a significantly higher overall cancer incidence in younger NF1 patients ( $<50 \mathrm{yr}$ old), with a standardized incidence ratio (SIR) of $6.5(95 \% \mathrm{Cl}$ 4.1-9.8, $P<0.0001)$. The SIR for all age groups with NF1 is $2.7(95 \% \mathrm{Cl} 1.9-3.7, P<$ 0.0001) when compared with the general population (Walker et al. 2006). An overall increase in mortality in NF1 patients also has been reported, with life expectancy reduced by 8-15 yr (Sørensen et al. 1986; Rasmussen et al. 2001; Evans et al. 2011). Although there are other complications, cancer is the most common cause of death in NF1 patients (Rasmussen and Friedman 2000). Potential relationships between variable clinical features in NF1 such as cancer predisposition, and particular mutations in NF1 are difficult to resolve, in part because some genetic variants such as the one described here are exceptionally rare.

MBC is comprised of a heterogeneous group of aggressive breast cancers made up of glandular epithelial, mixed nonglandular epithelial, or mesenchymal cell types (Greenberg et al. 2004; Natsiopoulos et al. 2007; Toumi et al. 2011). Classification of MBC into five subtypes has been proposed, including matrix-producing carcinoma, squamous cell carcinoma, spindle cell carcinoma, carcinosarcoma, and metaplastic carcinoma with osteoclastic giant 
cells (Luini et al. 2007). However, standardized pathological classification of MBC remains problematic because of the rarity of the disease, its histologic diversity, and a lack of a consensus about an appropriate classification system.

Several prior studies have described a possible association between breast cancer and NF1. An increased incidence of breast cancer in women with NF1 was reported, with a SIR of 2.9-5.2 compared to the general population (Sharif et al. 2007; Madanikia et al. 2012; Wang et al. 2012). Four additional studies demonstrated a much higher incidence of breast cancer in younger patients with NF1, with an elevated SIR of 4.0-8.8 in women $<50 \mathrm{yr}$ of age compared to $0.59-2.8$ in women $>50 \mathrm{yr}$ of age (Walker et al. 2006; Madanikia et al. 2012; Wang et al. 2012; Seminog and Goldacre 2015). The rate of breast cancer-related death in NF1 patients has been reported as a proportionate mortality ratio of 3.5 (95\% Cl, 1.3-7.7) (Evans et al. 2011). A case report described somatic LOH at NF1 in a 23-yr-old patient diagnosed with NF1 who developed infiltrating ductal breast carcinoma (Güran and Safali 2005).

Focusing on MBC arising in the context of NF1, we performed a detailed literature search and identified seven previously published papers describing breast cancers with features of MBC occurring in eight NF1 patients (Table 4; Malas et al. 1995; Nakamura et al. 1998; Natsiopoulos et al. 2007; Hegyi et al. 2009; Nogimori et al. 2014; Vivas et al. 2014; Chaudhry et al. 2015). None of these included molecular genetics or genomics analysis. We tabulated the patient demographics, clinical presentation, histology, stage, development of metastatic disease, follow-up, and outcomes as presented in these prior case reports (Table 4). All of the previously reported NF1 patients with MBC were female, with a mean age of $56.6 \mathrm{yr}$ (range from 41 to $76 \mathrm{yr}$ ). In papers that described clinical features on presentation, all patients presented with a large palpable breast mass. Half of them delayed seeking medical attention because they attributed their breast mass to the underlying NF1 disease. On biopsy, histological features of glandular epithelial cells and mixed nonglandular epithelial or mesenchymal cell types were described in all patients. The majority presented with an advanced tumor stage correlating with a T3 lesion, similar to the patient described here. Given that there is no universally accepted way to classify MBC, these previously published co-occurrences of MBC and NF1 were based on several different classifications. Only three of the previously reported cases were classified at the time as MBC. These findings emphasize the need for a standardized MBC classification method to prevent potential inappropriate diagnosis and treatment of this aggressive tumor subtype, as well as its underreporting.

The Cancer Genome Atlas Network (TCGA) cataloged nine genes in which germline genetic variants can predispose to breast cancer (i.e., ATM, BRCA1, BRCA2, BRIP1, CHEK2, NBN, PTEN, RAD51C, and TP53) (The Cancer Genome Atlas Network 2012). We identified germline SNVs in two of these genes in the patient (i.e., ATM and BRCA2). Of the four germline SNVs detected in ATM, one was a missense variant. However, it is relatively common, as its population allele frequency is 0.067 , thereby reducing the likelihood that it exerts a functional impact. The other SNVs in ATM were located in introns or were synonymous, respectively. Thus we conclude that germline SNVs in ATM did not contribute significantly to the patient's breast cancer pathogenesis. As described above, of the patient's six germline SNVs in BRCA2, one was a rare missense variant, so we included it in our list of functional SNV candidates identified here. However, it is designated as a benign variant in the ClinVar database. In addition, although it underwent LOH, its VAF decreased in the tumor. This suggested that any potential functional impact in predisposing to cancer formation was reduced by somatic $\mathrm{LOH}$ in the tumor.

The TCGA consortium also described 35 genes that are recurrently disrupted by somatic mutations in breast cancer, so they are considered to be strong candidates as breast cancercausing driver genes (The Cancer Genome Atlas Network 2012). Of the five genes disrupted 


\begin{tabular}{|c|c|c|c|c|c|c|c|c|c|}
\hline \multirow[b]{2}{*}{ Authors } & \multirow{2}{*}{$\begin{array}{c}\text { Age } \\
(y r)\end{array}$} & \multirow{2}{*}{$\begin{array}{l}\text { Presenting } \\
\text { complaint }\end{array}$} & \multirow{2}{*}{$\begin{array}{l}\text { Breast cancer histology; pathologic } \\
\text { classification }\end{array}$} & \multicolumn{3}{|c|}{ Stage } & \multirow{2}{*}{$\begin{array}{l}\text { Developed } \\
\text { distant } \\
\text { metastasis }\end{array}$} & \multirow{2}{*}{$\begin{array}{l}\text { Follow-up } \\
\text { (months) }\end{array}$} & \multirow[b]{2}{*}{ Outcome } \\
\hline & & & & $\mathrm{T}$ & $\mathrm{N}$ & $M$ & & & \\
\hline $\begin{array}{l}\text { Malas et al. } \\
1995\end{array}$ & 75 & $\begin{array}{l}\text { 6-cm left } \\
\text { breast } \\
\text { lump } \times 1 \mathrm{yr} \text {. }\end{array}$ & $\begin{array}{l}\text { Tubular carcinoma and spindle cell } \\
\text { tumor; high-grade malignant } \\
\text { schwannoma }\end{array}$ & 3 & 0 & 0 & No & 24 & Alive \\
\hline $\begin{array}{l}\text { Nakamura et al. } \\
1998\end{array}$ & $\begin{array}{l}44 \\
58\end{array}$ & a & $\begin{array}{l}\text { Spindle cell carcinoma } \\
\text { Squamous cell carcinoma }\end{array}$ & $\begin{array}{l}3 \\
2\end{array}$ & $\begin{array}{l}0 \\
0\end{array}$ & $\begin{array}{l}0 \\
0\end{array}$ & a & $\begin{array}{r}9 \\
76\end{array}$ & $\begin{array}{l}\text { Death } \\
\text { Alive }\end{array}$ \\
\hline $\begin{array}{l}\text { Chaudhry et al. } \\
2005\end{array}$ & 46 & $\begin{array}{l}\text { 8-cm left } \\
\text { breast mass }\end{array}$ & $\begin{array}{l}\text { Breast carcinoma with squamous } \\
\text { differentiation; metaplastic breast } \\
\text { carcinoma }\end{array}$ & 3 & 1 & 0 & No & 12 & Alive \\
\hline $\begin{array}{l}\text { Natsiopoulos } \\
\text { et al. } 2007\end{array}$ & 60 & $\begin{array}{l}\text { 9-cm left } \\
\text { breast } \\
\text { lump } \geq 6 \\
\text { mo }\end{array}$ & $\begin{array}{l}\text { Sarcomatous mesenchymal tumor with } \\
\text { foci of invasive ductal carcinoma; } \\
\text { carcinosarcoma }\end{array}$ & 2 & 0 & 0 & No & 30 & Alive \\
\hline $\begin{array}{l}\text { Hegyi et al. } \\
2009\end{array}$ & 41 & $\begin{array}{l}\text { 6-cm right } \\
\text { breast mass }\end{array}$ & $\begin{array}{l}\text { Tumor with glandular epithelial cells, } \\
\text { tubular structures, and spindle cells; } \\
\text { malignant myoepithelioma }\end{array}$ & 3 & 0 & 0 & a & a & a \\
\hline $\begin{array}{l}\text { Vivas et al. } \\
2014\end{array}$ & 53 & a & $\begin{array}{l}\text { Invasive ductal carcinoma with areas of } \\
\text { squamous differentiation, } \\
\text { hemangiopericytic pattern, and } \\
\text { osteoclast-like cells; metaplastic breast } \\
\text { carcinoma }\end{array}$ & & $a$ & & Yes & 7 & Death \\
\hline $\begin{array}{l}\text { Nogimori et al. } \\
2014\end{array}$ & 76 & $\begin{array}{l}\text { 7-cm left } \\
\text { breast } \\
\text { mass } \times 3 \mathrm{mo}\end{array}$ & $\begin{array}{l}\text { Sarcoma-like structures of highly atypical } \\
\text { spindle cells, glandular and sheet-like } \\
\text { epithelial structures; spindle cell } \\
\text { carcinoma }\end{array}$ & 4 & 3 & 0 & a & a & a \\
\hline $\begin{array}{l}\text { Suarez-Kelly } \\
\text { (this report) }\end{array}$ & 41 & $\begin{array}{l}\text { 6.3-cm right } \\
\text { breast mass }\end{array}$ & $\begin{array}{l}\text { Breast carcinoma with squamous } \\
\text { differentiation; metaplastic breast } \\
\text { carcinoma }\end{array}$ & 3 & 1 & 0 & Yes & 6 & Alive \\
\hline
\end{tabular}

We conducted a comprehensive literature review of MBC in NF1, published in the English language. Listed here are findings from seven previously published case reports (left) describing clinical features of eight breast cancer patients, including age of presentation, presenting complaint, pathologic features, final TNM staging based on pathology, and clinical outcomes.

a Data not reported.

by somatic mutations in our patient, only one was included on this list (i.e., TP53), whereas the others were not (The Cancer Genome Atlas Network 2012).

In analyzing SNVs in the blood and/or tumor samples, we observed that some were strongly affected by somatic $\mathrm{LOH}$ in the tumor, resulting in the somatic gain or loss of the variant allele. We also described an instance of two immediately adjacent SNVs, where the germline SNV did not show a change in its VAF in the tumor, thereby excluding somatic $\mathrm{LOH}$ of the haplotype. In contrast, the somatic mutation showed a VAF $<50 \%$, suggesting that it occurred in a subclone of tumor cells or was caused by potential contamination of the tumor sample by normal germline DNA but was not attributable to somatic $\mathrm{LOH}$ of the haplotype at that locus.

Of the 339 germline SNVs identified here, we identified four with potential functional impacts because they each occur rarely in the general population and are predicted to have missense or splicing site mutations. However, of these, the only functional SNV candidate that underwent $\mathrm{LOH}$, whereby the VAF was increased in the tumor, was the NF1-causing SNV in NF1. This provided further corroboration that this SNV very likely caused the patient's clinical NF1 syndrome by loss of the wild-type allele and increased allelic imbalance of the 
deleterious variant. Therefore, we conclude that $\mathrm{LOH}$ at this $\mathrm{SNV}$ contributed to formation of her MBC.

We acknowledge potential limitations in our study. First, as is customary for case reports, we focused on molecular findings in only one patient, making statistical analysis or extrapolation to other individuals with MBC in NF1 difficult. Second, the method of targeted exome sequencing excludes the possible identification of numerous potential SNVs outside of the relatively small captured genomic region. In addition, because of well-known technical limitations of targeted exome sequencing data, we did not attempt to use the data to discover other forms of genetic variation such as copy-number gains or genomic structural variation. Thus, we speculate that the patient was very likely to harbor many other significant predisposing variants, both in her germline DNA and in the tumor, that were missed by this targeted method. Third, available clinical databases cataloging candidate variants still are limited and in general do not include information about $\mathrm{LOH}$. We note that predicted functional impacts and annotated clinical features of some SNVs miscalled their likely impacts. For example, the rare, NF1-causing SNV in NF1 (c.2709G >A) is listed as synonymous and benign in multiple databases. However, it was reported in two independent studies to create a cryptic splice donor site, thereby disrupting pre-mRNA splicing, deleting 44 amino acids, and causing the disease (Brinckmann et al. 2007; Wimmer et al. 2007). Detection of cryptic splice sites is technically challenging using bioinformatics tools and will require further experimental assessment and annotation of candidate SNVs. It is possible that other SNVs may exert functional impacts that we cannot identify currently. Finally, although we confirmed the disease-causing splicing variant in NF1 using three different molecular methods, we did not evaluate its impact by investigating disrupted NF1 transcript splicing or protein expression. Such studies would be far outside the scope of this molecular case report, in part because proper consent and additional samples were not available.

In summary, we have identified a synonymous variant in NF1 that has been reported previously to disrupt the gene's pre-mRNA splicing, likely causing her NF1. Somatic LOH at numerous germline SNVs, including the disease-causing SNV in NF1, was identified in the patient's tumor. This increase in the NF1 variant's allelic fraction could increase its deleterious functional impacts, thereby contributing to the patient's MBC. In addition, we described two somatic SNVs in TP53 and BRCA1 that are likely to have contributed to tumor formation. These findings emphasize that combined analysis of germline SNVs and matched tumor SNVs will benefit molecular genetics studies of cancer patients, so that potential predisposing genetic factors and $\mathrm{LOH}$ can be identified. Characterization of $\mathrm{LOH}$ (with loss of the reference allele) would help elucidate if and how particular candidate variants may contribute to tumor formation.

\section{METHODS}

\section{DNA Isolation and Analysis}

Informed consent was obtained from the patient to participate in an IRB-approved protocol at The Ohio State University, to identify cancer-related genetic mutations by targeted DNA sequencing of her tumor and blood.

The patient's tumor sample was evaluated by a pathologist to ensure that tumor content exceeded $60 \%$. A blood sample was used as a matched control. Genomic DNA was purified from the samples using a QIAGEN QIAamp DNA FFPE tissue kit and QIAGEN DNeasy kit respectively, according to the manufacturer's instructions, and was eluted in EB buffer. DNA quality was assessed using a Nanodrop 2000 spectrophotometer (Thermo Fisher) and Agilent TapeStation, and concentrations were measured using a Qubit 2.0 Fluorometer (Thermo Fisher). 
Approximately 50-200 ng each of genomic DNA samples with optical density 260/280 ratio of $>1.5$ were used to construct barcoded, next-generation sequencing libraries. A custom panel of $120 \mathrm{nt}$ DNA oligonucleotides was used to enrich for exon sequences of 279 genes with known cancer associations by hybrid capture (Supplemental Table 1). Adaptor and barcode were ligated, germline DNA samples (50 ng) and tumor DNA samples (200 ng) were hybridized to the capture baits, and libraries were amplified and then quantified using a KAPA library quantification kit. Sequencing $(2 \times 100$ paired-end reads) was performed using the Illumina MiSeq platform with v.2 chemistry, resulting in approximately 4.6 million reads for normal and 5.5 million reads for the tumor sample. A table summarizing the sequencing depth of coverage is provided in Supplemental Table 2.

To confirm potential NF1-causing variants, genomic DNA purified from a whole blood sample was sent to the University of Alabama-Birmingham medical genomics laboratory. After extraction of genomic DNA from the blood leukocytes, Haloplex (Agilent) sequencing was performed to screen for SNVs or small insertion/deletion (indel) variants in the NF1-causing genes NF1 and SPRED1. Also included were a template control and a positive (sensitivity) control to confirm assay detection of low-level mosaic mutations (VAF $>0.08$ ). The NF1-causing variant identified in NF1 exon 21 was confirmed by Sanger sequencing at the UAB clinical genetics laboratory.

\section{Sequencing Data Quality Control and Analysis}

Sequence reads generated by the MiSeq instrument were demultiplexed using bcl2fastq at default settings. Outputs were scored for per-base quality using FastQC software (http:// www.bioinformatics.bbsrc.ac.uk/projects/fastqc/) to define bases with $>\mathrm{Q} 20$ and $>\mathrm{Q} 30$ quality scores. Reads were aligned to the human genome reference assembly hg19 (GRCh37.p13), specifically to the 279 targeted genes (Supplemental Table 1) using BWAMEM v. 0.7.14 (Li and Durbin 2010). PicardTools v. 2.3.0 was used to identify duplicate reads (http://broadinstitute.github.io/picard/). We used the Genome Analysis Toolkit (GATK) v. 3.5 (RealignerTargetCreator and IndelRealigner) (McKenna et al. 2010) and PicardTools (FixMatelnformation) tools to realign sequence reads considering known insertions/deletions (indels) in the dbSNP file hg19 snp150 (dbSNP; https://cgsmd.isi.edu/dbsnpq/). We performed base quality recalibrations using GATK BaseRecalibrator and PrintReads functions. Read depths and uniformity of coverage across individual exons were assessed (Supplemental Table 2).

Germline variants were detected in the normal sample and at the corresponding position in the tumor using SAMtools mPileup v. 1.2. Both the depth of sequencing coverage and sequence data quality were evaluated by using this package with default settings. We considered SNVs to be germline variants only when they were detected in both the normal and tumor samples.

We used a modification of our previously published methods to identify somatic SNVs (Samorodnitsky et al. 2015). Somatic variants present in the tumor sample and not in the matched germline (blood) sample were identified using MuTect v. 1.1.7 (Cibulskis et al. 2013). False positives that were labeled by MuTect as "reject" were excluded. We required a minimum of $5 \% \mathrm{VAF}$ for each somatic variant.

\section{Assessment of Population Allele Frequencies and Potential Functional Impacts of Genetic Variants}

Population allele frequencies and potential functional impacts of particular germline SNVs were annotated using VEP release 90 from ENSEMBL (McLaren et al. 2016). To determine population allele frequencies, we accessed ENSEMBL database release 90, human reference assembly hg19, ClinVar (release 2017-06) at the National Center for Biotechnology 
Information (https://www.ncbi.nlm.nih.gov/clinvar/), dbSNP (v150), COSMIC (release 81), 1000 Genomes (phase 3), NHLBI-ESP (V2-SSA137), and gnomAD (r2.0 170228, exomes only) (Lek et al. 2016). For SNVs detected in BRCA1 and BRCA2, we accessed data from ClinVar (201706), ENIGMA from the BRCA data exchange consortium (http://enigmaconsortium.org), and the Breast Cancer Information Core (BIC) database (https://research.nhgri.nih.gov/bic/). We identified potential functional impacts of variants using VEP (v. 90) with add-on packages including PolyPhen (2.2.2), SIFT (5.2.2), MetaSVM, MutationAssessor, Condel, and CADD.

Somatic SNVs were annotated using ANNOVAR (McKenna et al. 2010). Their prior identification was checked using databases including ENSEMBL (http://www.ensembl.org), dbSNP snp150, Catalogue of Somatic Mutations in Cancer hg19 v. 68 (COSMIC; http://cancer.sanger.ac.uk/cosmic), and Cancer Driver Log (CanDL; http://candl.osu. edu/contact) (Damodaran et al. 2015).

To calculate the VAF for each SNV identified in the normal blood sample and/or matched tumor sample, we used a custom $R$ script to count individual aligned reads supporting a base call at a particular position in the targeted exome with $Q>30$.

\section{Systematic Review of the Literature}

We performed a systematic review of the English-language literature for associations between MBC and NF1 (Table 4). We searched all MEDLINE/PubMed databases from each database's inception to November 2015. Given the lack of a standardized pathologic classification of MBC, several different searches were performed for keyword identification in all fields. The search strategy included key terms "breast neoplasms" or "breast" and "cancer" and "neurofibromatosis" and "metaplastic" or "spindle cells" or "squamous cell" or "osteoclastic" or "giant cells" or "carcinosarcoma" or "sarcoma" or "matrix-producing." For an article to be included, it required the presentation of a patient with (i) a diagnosis of NF1 and (ii) a breast neoplasm with histologic classification of glandular epithelial cells and mixed nonglandular epithelial or mesenchymal cell types.

\section{ADDITIONAL INFORMATION}

\section{Data Deposition and Access}

The NF1-causing variant in exon 21 of NF1 at c.2709G>A, Chr 17: 29556342 has been deposited in the National Center for Biotechnology Information (NCBI) ClinVar database (https://www.ncbi.nlm.nih.gov/clinvar/), accession numbers SCV000680081 and RCV000415263. Raw sequencing data was submitted to dbGaP (https://www.ncbi.nlm.nih. gov/gap) and can be found under accession number phs001566.v1.p1.

\section{Ethics Statement}

Written informed consent was obtained from the patient to participate in a cancer genetics study approved by The Ohio State University Cancer Biomedical Sciences Institutional Review Board.

\section{Acknowledgments}

We thank the patient described in this report for providing informed consent to participate in our genetics study; the medical genomics laboratory at the University at AlabamaBirmingham for performing screens for variants in NF1 and SPRED1; and Dr. Amanda Toland and Leigha Senter (The Ohio State University) for discussions about potential clinical implications of a BRCA2 variant. 
Competing Interest Statement

The authors have declared no competing interest.

Received September 27, 2017; accepted in revised form January 30, 2018.

\section{Author Contributions}

L.P.S.-K. and W.E.C. conceptualized this case report; L.P.S.-K., J.W.R., S.R., M.R., A.S., and W.E.C. generated sequencing data; K.A., J.W.R., E.S., and D.E.S. analyzed sequencing data; L.P.S.-K. and D.E.S. wrote the paper; L.P.S.-K., D.E.S., and W.E.C. edited the paper. All authors approved the paper.

\section{Funding}

This work was supported in part by the National Institutes of Health grants T32 5T32CA090223-12 (L.P.S.-K.), P01 CA95426 (W.E.C.), R50 CA211533 (K.A.), P30 CA016058 (K.A. and D.E.S.); The Ohio State University Comprehensive Cancer Center (K.A., S.R., D.E.S., W.E.C.); and the University of Texas MD Anderson Cancer Center (K.A. and D.E.S.).

\section{REFERENCES}

Barnes PJ, Boutilier R, Chiasson D, Rayson D. 2005. Metaplastic breast carcinoma: clinical-pathologic characteristics and HER2/neu expression. Breast Cancer Res Treat 91: 173-178.

Boyd KP, Korf BR, Theos A. 2009. Neurofibromatosis type 1. J Am Acad Dermatol 61: 1-14.

Brasfield RD, Das Gupta TK. 1972. Von Recklinghausen's disease: a clinicopathological study. Ann Surg 175: 86-104.

Brems H, Chmara M, Sahbatou M, Denayer E, Taniguchi K, Kato R, Somers R, Messiaen L, De Schepper S, Fryns JP, et al. 2007. Germline loss-of-function mutations in SPRED1 cause a neurofibromatosis 1-like phenotype. Nat Genet 39: 1120-1126.

Brems H, Pasmant E, Van Minkelen R, Wimmer K, Upadhyaya M, Legius E, Messiaen L. 2012. Review and update of SPRED1 mutations causing Legius syndrome. Hum Mutat 33: 1538-1546.

Brinckmann A, Mischung C, Bassmann I, Kuhnisch J, Schuelke M, Tinschert S, Nurnberg P. 2007. Detection of novel NF1 mutations and rapid mutation prescreening with Pyrosequencing. Electrophoresis 28: 4295-4301.

Campos B, Balmaña J, Gardenyes J, Valenzuela I, Abad O, Fàbregas P, Volpini V, Díez O. 2013. Germline mutations in NF1 and BRCA1 in a family with neurofibromatosis type 1 and early-onset breast cancer. Breast Cancer Res Treat 139: 597-602.

Chaudhry US, Yang L, Askeland RW, Fajardo LL. 2015. Metaplastic breast cancer in a patient with neurofibromatosis. J Clin Imaging Sci 5: 1-4.

Cibulskis K, Lawrence MS, Carter SL, Sivachenko A, Jaffe D, Sougnez C, Gabriel S, Meyerson M, Lander ES, Getz G. 2013. Sensitive detection of somatic point mutations in impure and heterogeneous cancer samples. Nat Biotechnol 31: 213-219.

Cooper CL, Karim RZ, Selinger C, Carmalt H, Lee CS, O'Toole SA. 2013. Molecular alterations in metaplastic breast carcinoma. J Clin Pathol 66: 522-528.

Damodaran S, Miya J, Kautto E, Zhu E, Samorodnitsky E, Datta J, Reeser JW, Roychowdhury S. 2015. Cancer Driver Log (CanDL): catalog of potentially actionable cancer mutations. J Mol Diagn 17: 554-559.

Deng G, Lu Y, Zlotnikov G, Thor AD, Smith HS. 1996. Loss of heterozygosity in normal tissue adjacent to breast carcinomas. Science 274: 2057-2059.

Evans DGR, O'Hara C, Wilding A, Ingham SL, Howard E, Dawson J, Moran A, Scott-Kitching V, Holt F, Huson SM. 2011. Mortality in neurofibromatosis 1: in North West England: an assessment of actuarial survival in a region of the UK since 1989. Eur J Hum Genet 19: 1187-1191.

Ferner RE, Huson SM, Thomas N, Moss C, Willshaw H, Evans DG, Upadhyaya M, Towers R, Gleeson M, Steiger $C$, et al. 2007. Guidelines for the diagnosis and management of individuals with neurofibromatosis 1. J Med Genet 44: 81-88.

Gao J, Aksoy BA, Dogrusoz U, Dresdner G, Gross B, Sumer SO, Sun Y, Jacobsen A, Sinha R, Larsson E, et al. 2013. Integrative analysis of complex cancer genomics and clinical profiles using the cBioPortal. Sci Signal 6: pl1.

Greenberg D, McIntyre H, Boerre T. 2004. Metaplastic breast cancer. Australas Radiol 48: 243-247.

Güran S, Safali M. 2005. A case of neurofibromatosis and breast cancer: loss of heterozygosity of NF1 in breast cancer. Cancer Genet Cytogenet 156: 86-88. 
Hegyi L, Thway K, Newton R, Osin P, Nerurkar A, Hayes AJ, Fisher C. 2009. Malignant myoepithelioma arising in adenomyoepithelioma of the breast and coincident multiple gastrointestinal stromal tumours in a patient with neurofibromatosis type 1. J Clin Pathol 62: 653-655.

Huson SM, Harper PS, Compston DA. 1988. Von Recklinghausen neurofibromatosis. A clinical and population study in south-east Wales. Brain 111: 1355-1381.

Kerangueven F, Noguchi T, Coulier F, Allione F, Wargniez V, Simony-Lafontaine J, Longy M, Jacquemier J, Sobol H, Eisinger $F$, et al. 1997. Genome-wide search for loss of heterozygosity shows extensive genetic diversity of human breast carcinomas. Cancer Res 57: 5469-5474.

Khalil J, Afif M, Elkacemi H, Benoulaid M, Kebdani T, Benjaafar N. 2015. Breast cancer associated with neurofibromatosis type 1: a case series and review of the literature. J Med Case Rep 9: 61.

Korf BR. 2013. Neurofibromatosis. In Handbook of clinical neurology (ed. Dulac O, et al.), Vol. 111, pp. 333-340. Elsevier, New York.

Kryukov GV, Pennacchio LA, Sunyaev SR. 2007. Most rare missense alleles are deleterious in humans: implications for complex disease and association studies. Am J Hum Genet 80: 727-739.

Lakshmaiah KC, Kumar AN, Purohit S, Viveka BK, Rajan KR, Zameer MAL, Namitha P, Saini ML, Azim HA Jr, Saini KS. 2014. Neurofibromatosis type I with breast cancer: not only for women! Hered Cancer Clin Pract 12: 5.

Larson PS, Schlechter BL, de las Morenas A, Garber JE, Cupples LA, Rosenberg CL. 2005. Allele imbalance, or loss of heterozygosity, in normal breast epithelium of sporadic breast cancer cases and BRCA1 gene mutation carriers is increased compared with reduction mammoplasty tissues. J Clin Oncol 23: 8613-8619.

Lee J, Wang J, Torbenson M, Lu Y, Liu QZ, Li S. 2010. Loss of SDHB and NF1 genes in a malignant phyllodes tumor of the breast as detected by oligo-array comparative genomic hybridization. Cancer Genet Cytogenet 196: 179-183.

Lee H, Jung SY, Ro JY, Kwon Y, Sohn JH, Park IH, Lee KS, Lee S, Kim SW, Kang HS, et al. 2012. Metaplastic breast cancer: clinicopathological features and its prognosis. J Clin Pathol 65: 441-446.

Lek M, Karczewski KJ, Minikel EV, Samocha KE, Banks E, Fennell T, O’Donnell-Luria AH, Ware JS, Hill AJ، Cummings BB, et al. 2016. Analysis of protein-coding genetic variation in 60,706 humans. Nature 536: 285-291.

Li H, Durbin R. 2010. Fast and accurate long-read alignment with Burrows-Wheeler transform. Bioinformatics 26: 589-595.

Luini A, Aguilar M, Gatti G, Fasani R, Botteri E, Brito JA, Maisonneuve P, Vento AR, Viale G. 2007. Metaplastic carcinoma of the breast, an unusual disease with worse prognosis: the experience of the European Institute of Oncology and review of the literature. Breast Cancer Res Treat 101: 349-353.

Madanikia SA, Bergner A, Ye X, O'Neill Blakeley J. 2012. Increased risk of breast cancer in women with NF1. Am J Med Genet A 158A: 3056-3060.

Maertens O, Prenen H, Debiec-Rychter M, Wozniak A, Sciot R, Pauwels P, De Wever I, Vermeesch JR, de Raedt T, De Paepe A, et al. 2006. Molecular pathogenesis of multiple gastrointestinal stromal tumors in NF1 patients. Hum Mol Genet 15: 1015-1023.

Malas S, Krawitz HE, Sur RK, Uijs RR, Nayler SJ, Levin CV. 1995. Von Recklinghausen's disease associated with a primary malignant schwannoma of the breast. J Surg Oncol 59: 273-275.

McKenna A, Hanna M, Banks E, Sivachenko A, Cibulskis K, Kernytsky A, Garimella K, Altshuler D, Gabriel S, Daly M, et al. 2010. The Genome Analysis Toolkit: a MapReduce framework for analyzing next-generation DNA sequencing data. Genome Res 20: 1297-1303.

McLaren W, Gil L, Hunt SE, Riat HS, Ritchie GR, Thormann A, Flicek P, Cunningham F. 2016. The ensembl variant effect predictor. Genome Biol 17: 122

Mendes-Pereira AM, Sims D, Dexter T, Fenwick K, Assiotis I, Kozarewa I, Mitsopoulos C, Hakas J, Zvelebil M, Lord CJ, et al. 2012. Genome-wide functional screen identifies a compendium of genes affecting sensitivity to tamoxifen. Proc Natl Acad Sci 109: 2730-2735.

Messiaen L, Yao S, Brems H, Callens T, Sathienkijkanchai A, Denayer E, Spencer E, Arn P, BabovicVuksanovic D, Bay C, et al. 2009. Clinical and mutational spectrum of neurofibromatosis type 1-like syndrome. JAMA 302: 2111-2118.

Nakamura M, Tangoku A, Kusanagi H, Oka M, Suzuki T. 1998. Breast cancer associated with Recklinghausen's disease: report of a case. Nihon Geka Hokan 67: 3-9.

Natsiopoulos I, Chatzichristou A, Stratis I, Skordalaki A, Makrantonakis N. 2007. Metaplastic breast carcinoma in a patient with von Recklinghausen's disease. Clinical Breast Cancer 7: 573-575.

Nogimori M, Yokota K, Sawada M, Matsumoto T, Kono M, Akiyama M. 2014. Spindle cell carcinoma of the breast in a patient with neurofibromatosis type 1. Eur J Dermatol 24: 397-398.

Rasmussen SA, Friedman JM. 2000. NF1 gene and neurofibromatosis 1. A J Epidemiol 151: 33-40.

Rasmussen SA, Yang Q, Friedman JM. 2001. Mortality in neurofibromatosis 1: an analysis using U.S. death certificates. Am J Hum Genet 68: 1110-1118. 
Roy C, Choudhury KB, Saha A, Bag S. 2014. Metaplastic carcinoma of the breast: a case report with review of literature. Ind J Cancer 51: 381-383.

Salemis NS, Nakos G, Sambaziotis D, Gourgiotis S. 2010. Breast cancer associated with type 1 neurofibromatosis. Breast Cancer 17: 306-309.

Samorodnitsky E, Jewell BM, Hagopian R, Miya J, Wing MR, Lyon E, Damodaran S, Bhatt D, Reeser JW, Datta J, et al. 2015. Evaluation of hybridization capture versus amplicon-based methods for whole-exome sequencing. Hum Mutat 36: 903-914.

Seminog OO, Goldacre MJ. 2015. Age-specific risk of breast cancer in women with neurofibromatosis type 1. Br J Cancer 112: 1546-1548.

Sharif S, Moran A, Huson SM, Iddenden R, Shenton A, Howard E, Evans DGR. 2007. Women with neurofibromatosis 1 are at a moderately increased risk of developing breast cancer and should be considered for early screening. J Med Genet 44: 481-484.

Sobjanek M, Dobosz-Kawałko M, Michajłowski I, Pęksa R, Nowicki R. 2014. Segmental neurofibromatosis. Postepy Dermatol Alergol 31: 410-412.

Sørensen SA, Mulvihill JJ, Nielsen A. 1986. Long-term follow-up of von Recklinghausen neurofibromatosis. Survival and malignant neoplasms. N Engl J Med 314: 1010-1015.

Spearman AD, Sweet K, Zhou XP, McLennan J, Couch FJ, Toland AE. 2008. Clinically applicable models to characterize BRCA1 and BRCA2 variants of uncertain significance. J Clin Oncol 26: 5393-5400.

The Cancer Genome Atlas Network. 2012. Comprehensive molecular portraits of human breast tumors. Nature 490: 61-70.

Toumi Z, Bullen C, Tang ACS, Dalal N, Ellenbogen S. 2011. Metaplastic breast carcinoma: a case report and systematic review of the literature. Pathol Int 61: 582-588.

Uusitalo E, Kallionpää RA, Kurki S, Rantanen M, Pitkäniemi J, Kronqvist P, Härkönen P, Huovinen R, Carpen O, Pöyhönen $M$, et al. 2017. Breast cancer in neurofibromatosis type 1: overrepresentation of unfavourable prognostic factors. Br J Cancer 116: 211-217.

Vivas APM, Bomfin LE, Pinto CA, Nicolau UR, Alves FA. 2014. Oral metastasis of metaplastic breast carcinoma in a patient with neurofibromatosis 1. Case Rep Oncol Med 2014: 1-7.

Walker L, Thompson D, Easton D, Ponder B, Ponder M, Frayling I, Baralle D. 2006. A prospective study of neurofibromatosis type 1 cancer incidence in the UK. Br J Cancer 95: 233-238.

Wallace MD, Pfefferle AD, Shen L, McNairn AJ, Cerami EG, Fallon BL, Rinaldi VD, Southard TL, Perou CM, Schimenti JC. 2012. Comparative oncogenomics implicates the neurofibromin 1 gene (NF1) as a breast cancer driver. Genetics 92: 385-396.

Walther MM, Herring J, Enquist E, Keiser HR, Linehan WM. 1999. von Recklinghausen's disease and pheochromocytomas. J Urol 162: 1582-1586.

Wang X, Levin AM, Smolinski SE, Vigneau FD, Levin NK, Tainsky MA. 2012. Breast cancer and other neoplasms in women with neurofibromatosis type 1: a retrospective review of cases in the Detroit metropolitan area. Am J Med Genet Part A 158A: 3061-3064.

Wimmer K, Roca X, Beiglbock H, Callens T, Etzler J, Rao AR, Krainer AR, Fonatsch C, Messiaen L. 2007. Extensive in silico analysis of NF1 splicing defects uncovers determinants for splicing outcome upon $5^{\prime}$ splice-site disruption. Hum Mutat 28: 599-612.

Xu W, Yang X, Hu X, Li S. 2014. Fifty-four novel mutations in the NF1 gene and integrated analyses of the mutations that modulate splicing. Int J Mol Med 34: 53-60.

Yap YS, McPherson JR, Ong CK, Rozen SG, Teh BT, Lee AS, Callen DF. 2014. The NF1 gene revisited-from bench to bedside. Oncotarget 15: 5873-5892.

Zöller MET, Rembeck B, Odén A, Samuelson M, Angervall L. 1997. Malignant and benign tumors in patients with neurofibromatosis type 1 in a defined Swedish population. Cancer 79: 2125-2131. 


\section{COLD SPRING HARBOR Molecular Case Studies}

\section{Metaplastic breast cancer in a patient with neurofibromatosis type 1 and somatic loss of heterozygosity}

Lorena P. Suarez-Kelly, Keiko Akagi, Julie W. Reeser, et al.

Cold Spring Harb Mol Case Stud 2018, 4: a002352 originally published online February 15, 2018 Access the most recent version at doi: $10.1101 / \mathrm{mcs} .0002352$
Supplementary http://molecularcasestudies.cshlp.org/content/suppl/2018/02/15/mcs.a002352.D Material C1

References This article cites 62 articles, 11 of which can be accessed free at: http://molecularcasestudies.cshlp.org/content/4/2/a002352.full.html\#ref-list-1

License This article is distributed under the terms of the Creative Commons Attribution-NonCommercial License, which permits reuse and redistribution, except for commercial purposes, provided that the original author and source are credited.

Email Alerting Receive free email alerts when new articles cite this article - sign up in the box at the Service top right corner of the article or click here. 